\title{
Two-Stage Depressurization in Gas Dissolution Foaming: The Production of Nanocellular Materials Free of Defects
}

\author{
Judith Martín-de León,* Victoria Bernardo, and Miguel Ángel Rodríguez-Pérez
}

\begin{abstract}
Nanocellular polymethylmethacrylate (PMMA) is produced through a newly proposed method, a two-stage depressurization in the gas dissolution foaming process. This method modifies the depressurization step and allows controlling the pressure during cell growth, avoiding this way, the appearance of micrometric defects in the produced cellular materials. Three grades of PMMA, as well as different production parameters, are tested in order to study their influence on the final materials. Moreover, cellular structures are compared with those obtained with a one-stage depressurization process. Additionally, this work analyzes the foaming mechanisms taking place during the production of nanocellular materials.
\end{abstract}

\section{Introduction}

Nanocellular polymers were born around ten years ago as an attempt to improve the properties of microcellular materials. In general terms, nanocellular polymers are those presenting cells in the nanometric scale and cell nucleation densities higher than $10^{13}$ nuclei $\mathrm{cm}^{-3} \cdot{ }^{[1,2]}$

Since its discovery, nanocellular materials have been produced by following multiple strategies, such as phase separation techniques, ${ }^{[3,4]}$ templating and imprinting techniques, ${ }^{[5-9]}$ or the most common one, gas dissolution foaming technique, ${ }^{[10]}$ and therefore, a broad range of results have been obtained. Thus, systems based on different polymeric matrices with a wide variety of cell sizes, cell nucleation densities, and relative densities have been obtained up to now. ${ }^{[11,12]}$

Among all these works in the literature, it is worth highlighting the results obtained by Costeux and Zhu through heterogeneous nucleation. On the one hand, by mixing polymethylmethacrylate (PMMA) and PMMA-co-EA (random copolymer $9 \%$ ethyl acrylate) with nanosilicas, they generated nanocellular materials with relative densities smaller than 0.23 and minimum cell sizes of $95 \mathrm{~nm}$. On the other hand, by mixing PMMA-co-EMA (random copolymer 50\% ethyl methacrylate) with polyhedral oligomeric silsesquioxane (POSS), they produced nanocellular materials with cell sizes ranging $100 \mathrm{~nm}$ combined with a relative density of $0.16 .^{[13]}$ Conversely, when

Dr. J. Martín-de León, Dr. V. Bernardo, Prof. M. A. Rodríguez-Pérez

Cellular Materials Laboratory (CellMat)

Universidad de Valladolid

47011, Valladolid, Spain

E-mail: jmadeleon@fmc.uva.es

The ORCID identification number(s) for the author(s) of this article can be found under https://doi.org/10.1002/mame.202000283.

DOI: $10.1002 /$ mame.202000283 working with homogeneous nucleation, the best results up to now were presented by Martín-de León et al. They produced a nanocellular material based on PMMA with cell sizes around $75 \mathrm{~nm}$ and a relative density of $0.24 .{ }^{[14]}$

Although the results reported so far are exciting, moving forward in this field (i.e., further reducing the density while keeping the cells in the nanoscale) is increasingly tricky, especially due to the lack of knowledge about the foaming mechanisms in nanocellular polymers. Besides that, in many cases, especially for low relative densities, the produced materials present internal defects and microcells which origin is not clear today. These defects are a potential source of failure in mechanical testing, and depending on their size and number, they could also affect other properties. For example, they could increase the thermal conductivity of the materials.

In this work, a new production approach, based on the one-step gas dissolution foaming process, ${ }^{[15,16]}$ has been developed for the production of nanocellular materials. In this new method, the depressurization step has been carried out in two stages. This new method has led to the removal of the microcellular defects observed in some nanocellular materials under certain production conditions. Moreover, the production parameters of this new processing route were modified to optimize them as a function of the viscosity of the polymer matrix.

Furthermore, the foaming mechanisms taking place during the production of PMMA based nanocellular materials have been studied. Thus, nucleation and cell growth were analyzed using a nucleation theory developed by Costeux et al. ${ }^{[17]}$ that allows understanding the physical phenomena taking place during the production of nanocellular materials.

\section{Experimental Section}

\subsection{Materials}

Three grades of PMMA were used in this work: $6 \mathrm{~N}, 7 \mathrm{~N}$, and V825T. All of them were supplied in the form of pellets by PLEXIGLAS Evonik Industries (Essen, Germany) in the case of grades $7 \mathrm{~N}$ and $6 \mathrm{~N}$, and by ALTUGLAS International (Colombes, France) in the case of V825T.

The density $(\rho)$ of the three PMMA grades was $1.19 \mathrm{~g} \mathrm{~cm}^{-3}$ (measured at $23{ }^{\circ} \mathrm{C}$ and $50 \% \mathrm{HR}$ ). Their glass transition temperature $\left(T_{\mathrm{g}}\right)$ measured through differential scanning calorimetry (Section 2.4.1), zero shear viscosity $\left(\eta_{0}\right)$, determined via 
Table 1. Glass transition temperature, zero shear viscosity, and melt flow index of the PMMAs under study.

\begin{tabular}{lccc}
\hline Material & $\begin{array}{c}\text { Class transition } \\
\text { temperature }\left[{ }^{\circ} \mathrm{C}\right]\end{array}$ & $\begin{array}{c}\text { Zero shear } \\
\text { viscosity[Pa s] }\end{array}$ & $\mathrm{MFI}[\mathrm{g} / 10 \mathrm{~min}]$ \\
\hline V825T (HV) & 114.4 & 7095 & 1.92 \\
7N (MV) & 109.3 & 3800 & 3.64 \\
$6 \mathrm{~N} \mathrm{(LV)}$ & 98.6 & 1587 & 8.20 \\
\hline
\end{tabular}

shear rheology (Section 2.4.2), and the melt flow index (MFI) (measured at $160{ }^{\circ} \mathrm{C}$ and $10 \mathrm{~kg}$ ) are shown in Table 1 . The three materials presented different characteristics being the PMMA V825T, the one with the highest viscosity (lowest MFI) accompanied with the highest glass transition temperature. On the contrary, the $6 \mathrm{~N}$ grade was the one with the lowest $T_{\mathrm{g}}$ and viscosity and the highest MFI.

According to these characteristics, from now on, they will be named as high viscosity (HV), medium viscosity (MV), and low viscosity (LV) polymethylmethacrylate.

Additionally, medical-grade $\mathrm{CO}_{2}$ ( $99.9 \%$ purity) was used as a blowing agent in the gas dissolution foaming process.

\subsection{Samples Production}

Solid samples of dimensions $20 \times 20 \times 4 \mathrm{~mm}^{3}$ were produced using compression molding.

First, pellets were dried at $80{ }^{\circ} \mathrm{C}$ for $4 \mathrm{~h}$ to remove the remaining moisture of the material. Afterward, they were compressed molded in a hot plate press from Remtex (Barcelona,
Spain) using a mold of a size of $68 \times 68 \times 4 \mathrm{~mm}^{3}$, and by following the next steps. First, the material was heated at $250{ }^{\circ} \mathrm{C}$ for 9 min. During these $9 \mathrm{~min}$, the material softened due to the used temperature, which was higher than its $T_{\mathrm{g}}$. Then, a pressure of $42 \mathrm{MPa}$ was applied for an additional minute while maintaining the temperature at $250^{\circ} \mathrm{C}$. In this step, the material took the shape of the cavity of the used mold. Finally, the sample was cooled down to room temperature for $4 \mathrm{~min}$ applying the same pressure.

The solid precursors were finally machined to the desired dimensions $\left(20 \times 20 \times 4 \mathrm{~mm}^{3}\right)$ for the foaming tests.

Cylindrical samples $1 \mathrm{~mm}$ thick for rheological measurements were produced following the same procedure.

\subsection{Foaming Tests}

Cellular materials were produced by following the method described in Figure 1a. This scheme represents a modified onestep gas dissolution foaming process.

One-step gas dissolution foaming process ${ }^{[18]}$ consists of three steps: saturation, depressurization, and finally, stabilization. First, the polymer is saturated with carbon dioxide $\left(\mathrm{CO}_{2}\right)$ under certain conditions of pressure $\left(P_{\text {sat }}\right)$ and temperature $\left(T_{\text {sat }}\right)$. Once the polymer is fully saturated, the gas is fast released up to reaching atmospheric pressure. This creates an instability in the gas-polymer system that results in phase-separation, that is, the creation of nucleation sites. Finally, samples are stabilized to prevent further foaming.

When the gas diffuses into the polymer, it triggers a reduction in the glass transition temperature of the polymer up to a

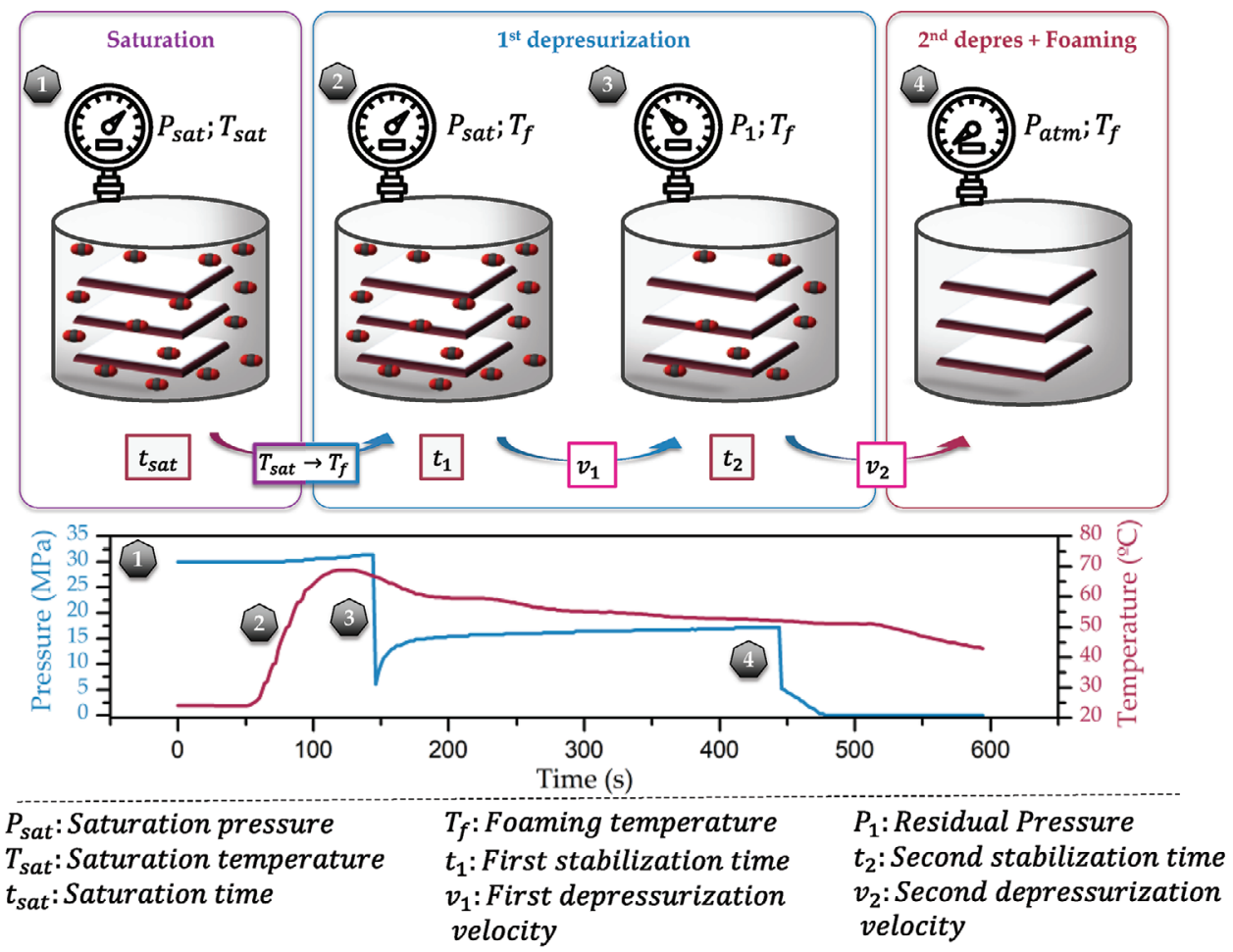

Figure 1. a) Scheme of the foaming process. b) Pressure and temperature as a function of time in a real experiment. 
Table 2. Foaming conditions of the different tests.

\begin{tabular}{|c|c|c|c|c|c|c|c|c|}
\hline Test & $P_{\text {sat }}[\mathrm{MPa}]$ & $\left.T_{\text {sat }}{ }^{\circ} \mathrm{C}\right]$ & $P_{1}[\mathrm{MPa}]$ & $\left.T_{\mathrm{f}} \mathrm{f}^{\circ} \mathrm{C}\right]$ & $t_{1}[\mathrm{~min}]$ & $v_{1}\left[\mathrm{MPa} \mathrm{s}^{-1}\right]$ & $t_{2}[\mathrm{~min}]$ & $v_{2}\left[\mathrm{MPa} \mathrm{s}^{-1}\right]$ \\
\hline $\mathrm{Tl}$ & 31 & 24 & 16.6 & 50 & 1 & 100 & 5 & 50 \\
\hline $\mathrm{T} 2$ & 31 & 24 & 6.8 & 50 & 1 & 100 & 5 & 0.2 \\
\hline $\mathrm{T} 3$ & 31 & 24 & - & 50 & 1 & 100 & 5 & - \\
\hline $\mathrm{T} 4$ & 31 & 24 & 17.3 & 100 & 1 & 100 & 5 & 50 \\
\hline T5 & 31 & 24 & 6.9 & 100 & 1 & 100 & 5 & 0.2 \\
\hline T6 & 31 & 24 & - & 100 & 1 & 100 & 5 & - \\
\hline
\end{tabular}

lower one, the so-called effective glass transition temperature $T_{\mathrm{geff}}{ }^{[19]}$ Then, if the saturation is performed at a $T_{\text {sat }}$ higher than $T_{g_{\text {eff }}}$ when the pressure is reduced, nucleation and growing occurs simultaneously. ${ }^{[18]}$

In this work, this one-step foaming process was modified as follows (Figure 1). First, saturation was performed under $P_{\text {sat }}$ and a saturation temperature smaller than the $T_{g_{\text {eff }}}$ of the polymer- $\mathrm{CO}_{2}$ system. After saturation, the temperature was increased up to a foaming temperature $\left(T_{\mathrm{f}}\right)$ (see Figure 1), higher than $T_{g_{\text {eff }}}$. This temperature was maintained during a stabilization time $t_{1}$, enough for the whole system to homogeneously reach the desired $T_{\mathrm{f}} . P_{\text {sat }}$ slightly increased during $t_{1}$ as a consequence of temperature increase. Then, a first partial depressurization up to a residual pressure $P_{1}$ was carried out at a depressurization velocity $v_{1}$. This situation was maintained during a time $t_{2}$, and finally, the gas was completely released in a second depressurization at a velocity $v_{2}$. Changes in pressure and temperature were monitored using a pressure sensor that reads the pressure value inside the pressure vessel and a thermocouple installed in the clamp heater around the vessel. Graphs, as shown in Figure 1b, were obtained for each experiment.

As $T_{\mathrm{f}}$ is higher than the $T_{\mathrm{g}_{\mathrm{eff}}}$ (near room temperature for those saturation conditions ${ }^{[20]}$ ), the polymer can grow when the pressure is completely released. The proposed method was named as two-stage depressurization foaming (TSD foaming).

Foaming tests were performed in a system that comprised a pressure vessel (model PARR 4681) provided by Parr Instrument Company (Moline, IL, USA). This one was connected to a pump (model SFT-10) supplied by Supercritical Fluid Technologies Inc. (Newark, DE, USA) responsible for providing the desired pressure to the system. Additionally, a clamp heater connected to a temperature controller CAL 3000 was used to adjust the temperature of the system. Finally, an electrovalve with $K_{\mathrm{v}}=1.1 \mathrm{~L} \mathrm{~min}^{-1}$ allowed to fast release the pressure.

Six different experiments were carried out in the described system by using the conditions shown in Table 2. Saturation conditions were fixed to be $31 \mathrm{MPa}$ and $24{ }^{\circ} \mathrm{C}$. According to the literature, these production parameters lead to solubilities higher than $30 \%$ for the three used materials that result in cells in the nanometric scale for all of them when the conventional two steps gas dissolution process is used. ${ }^{[21]}$ Then, a two-stage depressurization was carried out by using two different partial pressures $P_{1}, 17$ and $7 \mathrm{MPa}$. Additionally, this was done for two different foaming temperatures: 50 and $100{ }^{\circ} \mathrm{C}$ (higher enough than the $T_{\mathrm{g}_{\text {eff }}}$ at those conditions).

As it can be seen in Figure 2 and Table 2, the first pressure drop rate $\left(v_{1}\right)$ was equivalent for the two $P_{1}$ used (see Section 2.4.3 for the measurement method). In the first case, the pressure decreased from 31 to $5 \mathrm{MPa}$ leading to a depressurization rate of $v_{1}=100 \mathrm{MPa} \mathrm{s}^{-1}$ and then pressure recovered up to $P_{1}=17 \mathrm{MPa}$ (Figure 2a). In the second case, the pressure fell up to $5 \mathrm{MPa}$ and maintained its value being $P_{1}=7 \mathrm{MPa}$. This effect was achieved by changing the opening time of the electrovalve.

Stabilization times $t_{1}$ and $t_{2}$ were chosen to be 1 and $5 \mathrm{~min}$ for all the experiments.
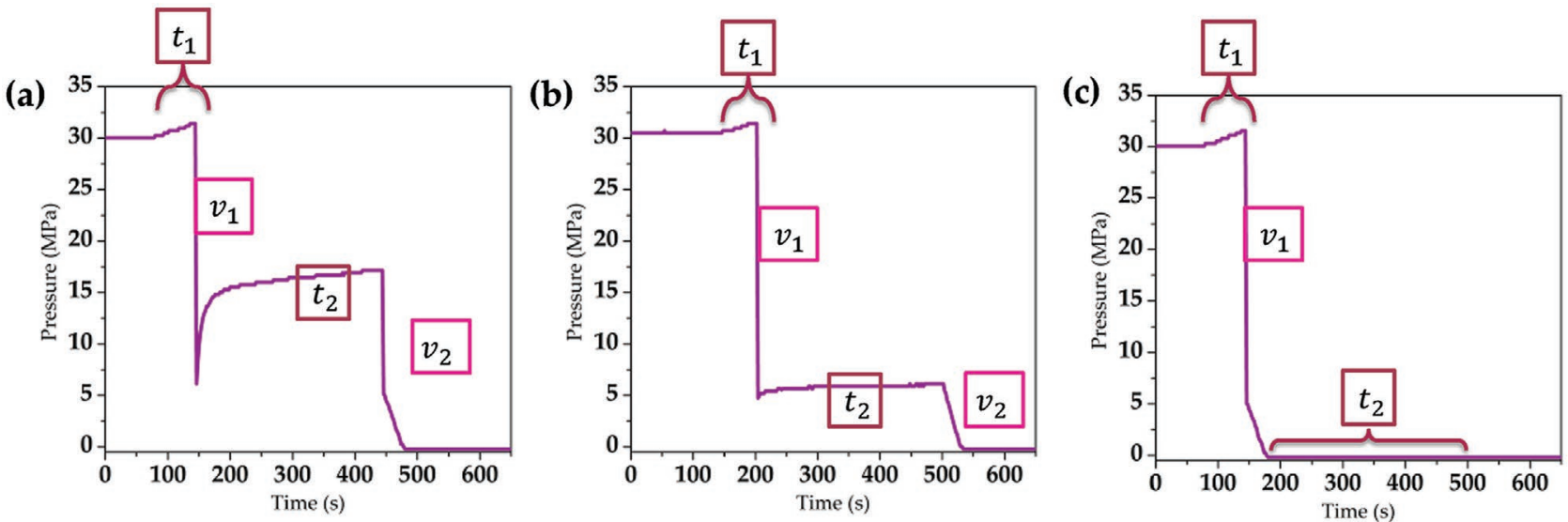

Figure 2. Examples of pressure versus time graphs for the partial depressurizations. a) For TSD foaming with high residual pressure (T1 and T4), b) for TSD foaming with low residual pressure (T2 and T5), and c) for OSD (T3 and T6). 
Two additional experiments (T3 and T6) were carried out with a single depressurization (one-stage depressurization foaming (OSD foaming)) with the aim of comparison (Figure 2c). In both of them, the pressure was completely released after $t_{1}$. After this time, samples were under atmospheric pressure and foaming temperature during $t_{2}=5 \mathrm{~min}$.

After the foaming time, all samples were removed from the pressure vessel in less than $30 \mathrm{~s}$ and then stabilized in water at room temperature.

\subsection{Characterization Techniques}

\subsubsection{Glass Transition Temperature}

A differential-scanning calorimeter model Metler DSC30, previously calibrated with indium, was used for the measurement of the glass transition temperature. With an amount of sample of $5 \mathrm{mg}$, the thermogram was obtained by heating from 20 to $160^{\circ} \mathrm{C}$ at $10^{\circ} \mathrm{C} \mathrm{min}^{-1}$. The value for the $T_{\mathrm{g}}$ was calculated as the mid-point of the drop in the thermogram that characterizes this transition.

\subsubsection{Polymers Rheology}

Shear rheology for the three used materials was carried out in a stress-controlled rheometer, AR 2000 EX, from TA Instruments. Zero shear viscosity, $\eta_{0}$, was determined through this technique.

Solid cylindrical samples were produced by compression molding (Section 2.2) to perform the measurements. Dynamic shear viscosity measurements were performed at $230^{\circ} \mathrm{C}$ under a nitrogen atmosphere and using a parallel plates geometry of $R=$ $25 \mathrm{~mm}$ in diameter and a fixed gap of $h=1 \mathrm{~mm}$. The angular frequency range was $0.01<w<100 \mathrm{rad} \mathrm{s}^{-1}$, and a strain of $6 \%$ was used. The zero shear viscosity was calculated as the value of the complex viscosity at low frequencies in the Newtonian plateau ${ }^{[22]}$ from the dynamic shear viscosity measurements.

\subsubsection{Depressurization Velocity Determination}

To accurately determine the depressurization velocity, pressure was recorded by using a sampling frequency of $0.001 \mathrm{~s}$. The depressurization velocity was considered as the slope of the first linear part of the graph (Figure 3).

\subsubsection{Density}

The density of solid samples $\left(\rho_{\mathrm{s}}\right)$ were measured through a gas pycnometer (Mod. AccuPyc II 1340) provided by Micromeritics, Norcross, GA, USA. The density of cellular materials $\rho_{\mathrm{f}}$ was measured by using the density determination kit of an AT261 Mettler-Toledo balance and considering the water displacement method, based on Archimedes' principle.

Before those measurements, cellular materials were polished to remove the solid outer skin (around $200 \mu \mathrm{m}$ were

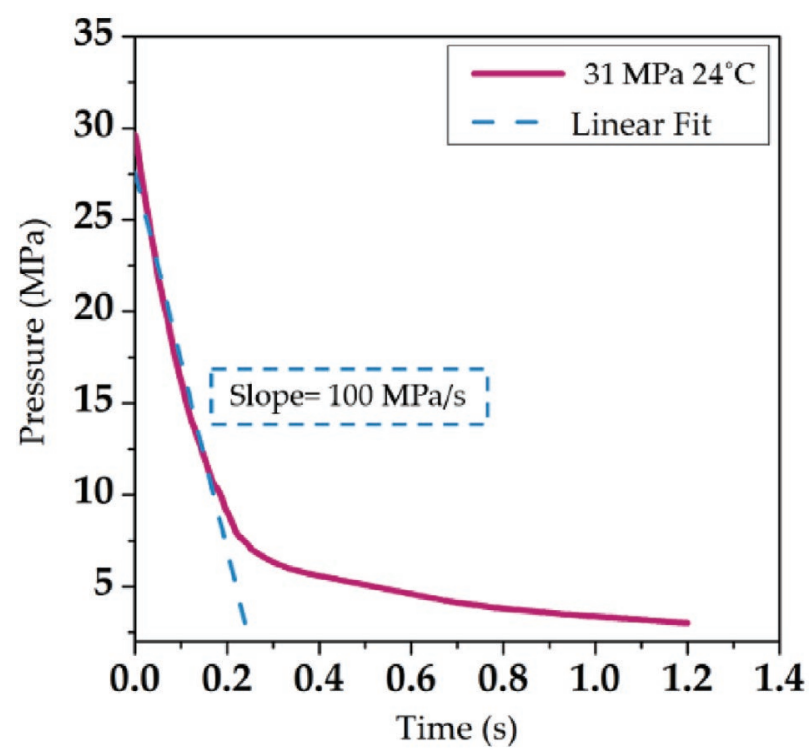

Figure 3. Example of pressure versus time graph in the moment of depressurization from 31 to $0 \mathrm{MPa}$.

removed from each side). Finally, the relative density $\rho_{\mathrm{r}}$ was calculated as the ratio between the cellular material density and the solid one.

\subsubsection{Scanning Electron Microscopy (SEM)}

Cellular structure was visualized using an ESEM Scanning Electron Microscope (FlexSEM1000 provided by Hitachi). In order to correctly visualize the samples, it was needed to fracture them in liquid nitrogen to preserve the original cellular structure. Then, they were coated with gold using a sputter coater (model SDC 005, Balzers Union, Balzers, Liechtenstein), and finally, they were visualized.

The cellular structure was analyzed using a software, based on ImageJ/FIJI. ${ }^{[23]}$ Thus, the cell size $(\varphi)$, the standard deviation of the cell size distribution (SD), the parameter $\mathrm{SD} / \varphi$, and the cell nucleation density $\left(N_{0}\right)$ were determined for each produced material. $N_{0}$ was calculated by following the Kumar's method ${ }^{[24]}$ Several samples for each material and each production condition were analyzed. The results shown in the next section are the mean values of that analysis.

\section{Results}

\subsection{Cellular Structure}

\subsubsection{External Appearance}

In order to analyze the internal structure of the produced cellular materials (homogeneity and defects), SEM images at low magnifications were taken as it is shown in Figure 4.

The first row in the image that means Figure $4 \mathrm{a}-\mathrm{c}$ corresponds to the materials produced in test T3, namely, by following the one depressurization process (OSD). The two 


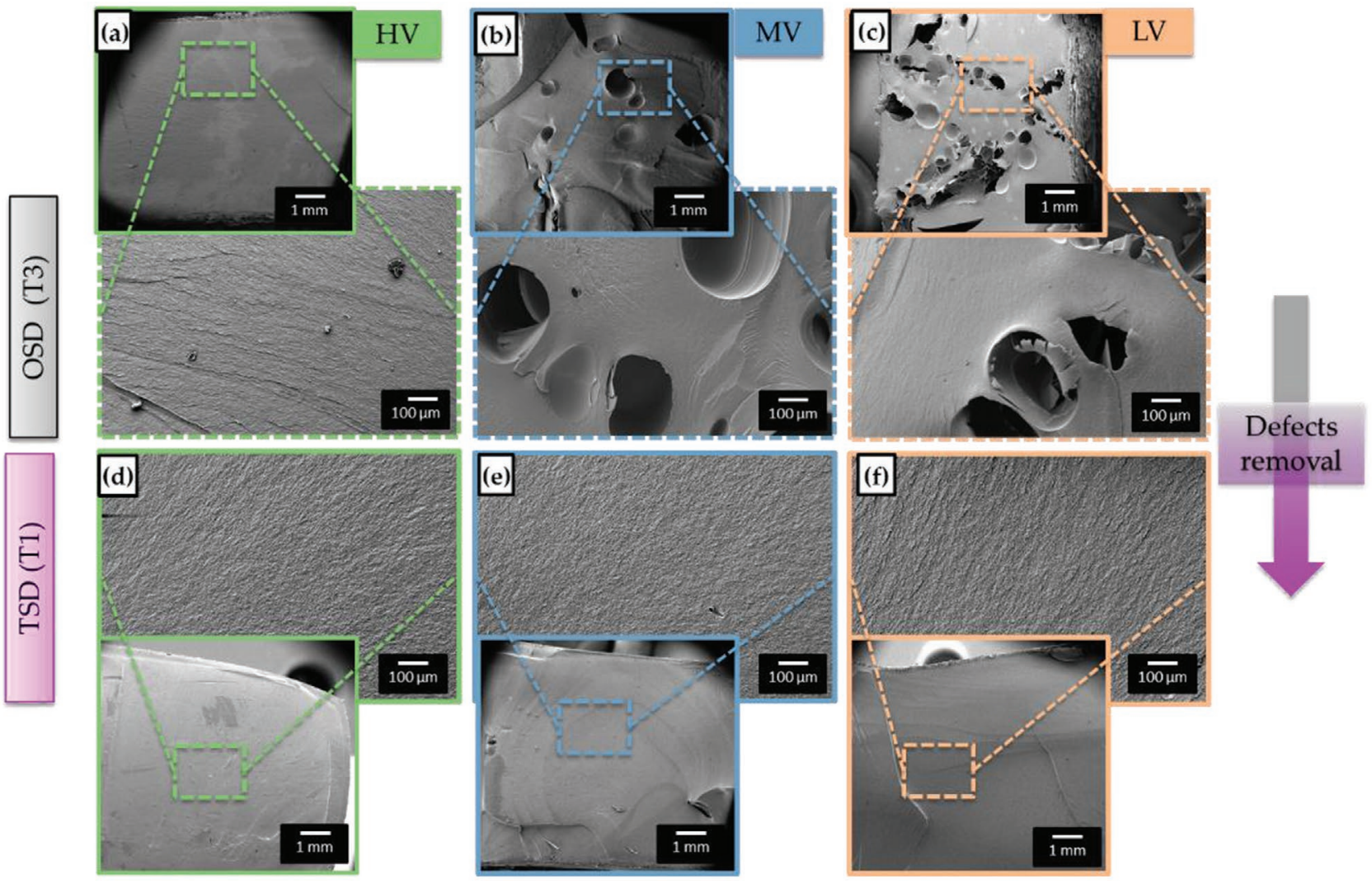

Figure 4. Low magnification SEM images for the visualization of defects. First row for materials produced in test T3: a) HV, b) MV, and c) LV. Second row for materials produced in $\mathrm{Tl}$ : d) $\mathrm{HV}$, e) MV, and f) LV.

PMMA with lower viscosities, MV, and LV, exhibit some big holes in the millimetric scale (Figure $4 \mathrm{~b}, \mathrm{c}$ ). When comparing these results to the structures obtained when foaming by following a two-stage depressurization (experiment T1), the mentioned defects disappear (Figure 4e,f). It is also interesting to point out that this behavior also depends on the used material; thus, the one with the highest viscosity can be produced free of defects following both production routes.

Similar results are obtained for the rest of the tests, so materials based on MV and LV fabricated in test T3 and T6 (via OSD) show large holes, while in the experiments (T1, T2, T4, and T5) (via TSD) the materials do not present any defects.

These findings can be justified through the foaming mechanisms that relate the foaming conditions with the cellular structure, as will be discussed in Section 3.2.

So herein, it can be concluded that the new proposed process (TSD foaming) makes it possible to produce nanocellular materials completely free of defects. On the other hand, this procedure also affects the nanocellular structure of the samples, as it is examined in the following section.

\subsubsection{Nanocellular Structure}

Figure 5 shows examples of the cellular structure of different produced materials comparing both production routes ODS and TDS.
All the produced cellular materials present cells in the nanometric scale independently on the used route. For the samples produced through the one depressurization process, a homogeneous cellular structure is found (Figure $5 a-c$ ). In those samples presenting defects (MV and $\mathrm{LV}$ ), the structure shown in Figure 5 corresponds to the area between the mentioned defects. On the other hand, samples produced through the defined new process (TSD) present a nanocellular structure as that shown in Figure $5 \mathrm{~d}-\mathrm{f}$ in the whole sample (after removing the solid skin). All the samples are homogeneous along its entire volume. This was proven by taking SEM images in different regions of the materials (see Section S2 and Figure S1 in the Supporting Information).

Although both processes lead to a nanocellular structure, there exists a clear difference between the structures for the three used PMMA. Figure 5 shows that the TSD process (second row) leads to larger cell sizes and lower cell nucleation densities independently on the PMMA viscosity. In order to in-depth analyze those changes, relative density, cell size, and cell nucleation density were measured and plotted as a function of the residual pressure $P_{1}$ for all the experiments (Figure 6). Experiments with $P_{1}=0 \mathrm{MPa}$ corresponds to one-depressurization foaming tests (T3 and T6).

It can be observed that both a change between a single depressurization process and a two depressurization one, as well as a change in the residual pressure, leads to changes in the cellular structure. 

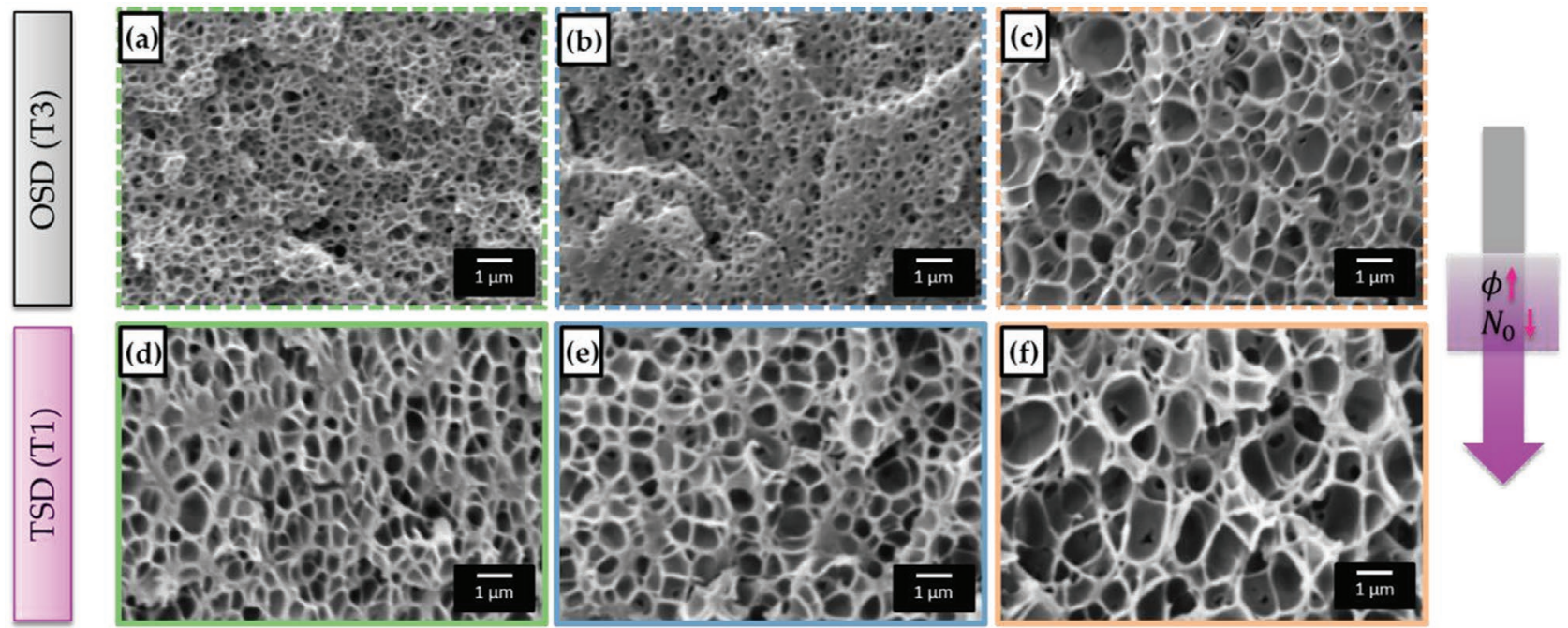

Figure 5. SEM images for the visualization of the internal nanocellular structure. First row for materials produced in test T3: a) HV, b) MV, and c) LV. Second row for materials produced in T1: d) HV, e) MV, and f) LV.

First of all, the effect of changing from a single depressurization to a partial one will be studied (arrows in Figure 6). Single depressurization leads to higher cell nucleation densities (Figure 6b,e) and smaller cell sizes (Figure 6c,f): the cell nucleation density decreases over half an order of magnitude, while the cell size almost doubles for the three materials.

Conversely, when studying the relative density, no clear effect is observed with the change of the process (Figure 6a,d)). For the LV, the density slightly increases, and for MV and HV, (a)

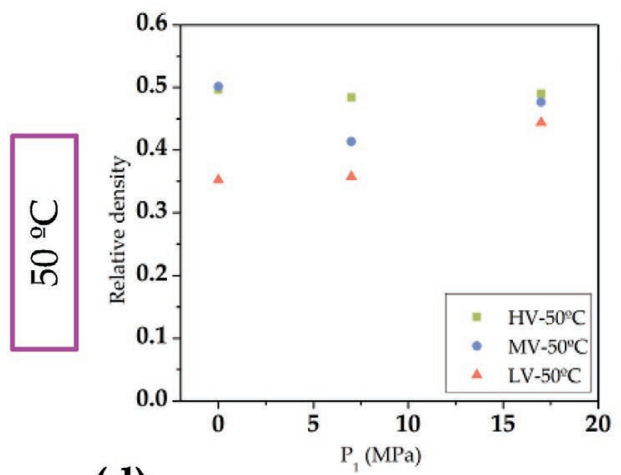

(d)

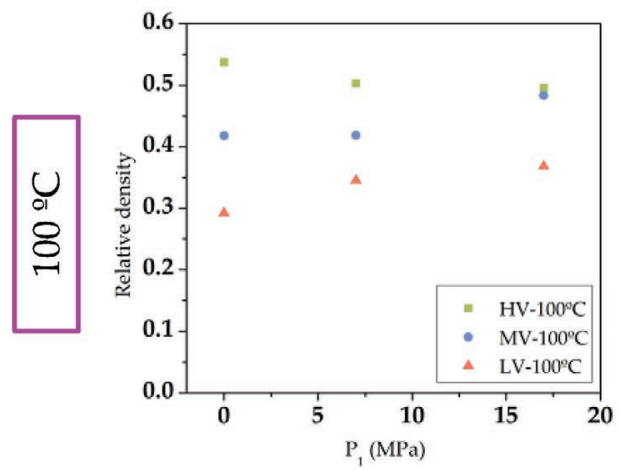

(b)

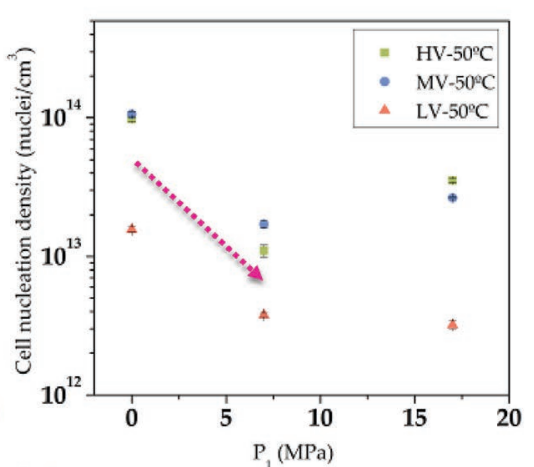

(e)

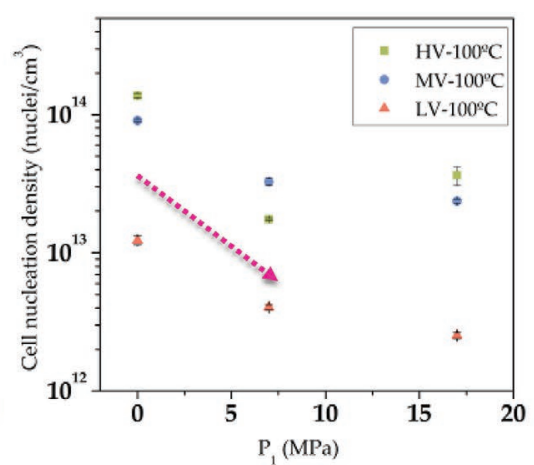

(c)

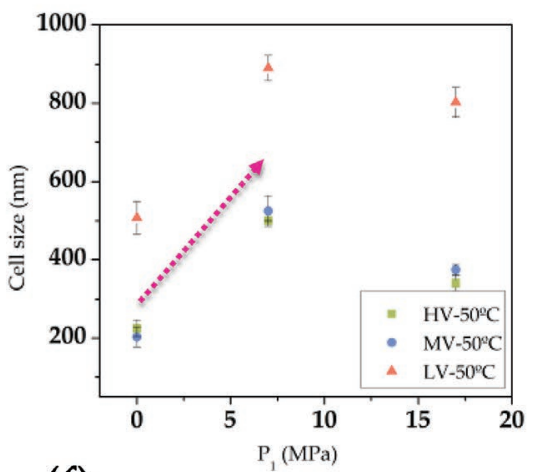

(f)

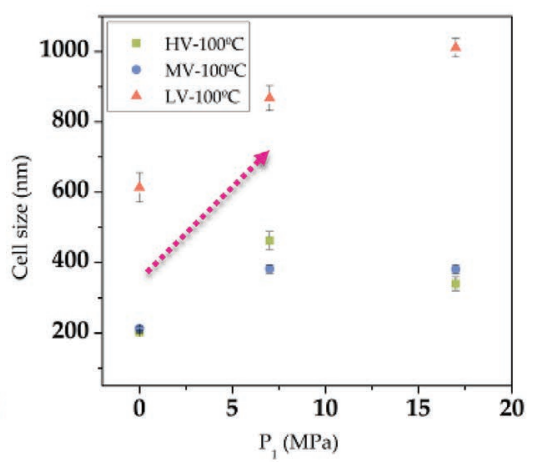

Figure 6. Graphs corresponding to $50{ }^{\circ} \mathrm{C}$ of foaming temperature: a) Relative density as a function of $P_{1}$. b) Cell nucleation density as a function of $P_{1}$. c) Cell size as a function of $P_{1}$. Graphs corresponding to $100{ }^{\circ} \mathrm{C}$ of foaming temperature: d) Relative density as a function of $P_{1}$. e) Cell nucleation density as a function of $P_{1}$. f) Cell size as a function of $P_{1}$. Arrows indicate the change of tendency between one-depressurization foaming process and two-stage depressurization process. 
slightly decreases. This fact is understandable because relative density depends on both the cell nucleation density and the cell size. This relationship is established through the following equation ${ }^{[24]}$

$\frac{1}{\rho_{\mathrm{r}}}=1+\left(\frac{N_{0} \phi^{3} \pi}{6}\right)$

So, while a decrease in the cell nucleation density increases the relative density, bigger cells lead to a smaller relative density. Therefore, results regarding $\rho_{\mathrm{r}}$ are the consequence of the competition between those two parameters. Overall, it could be stated that the $\rho_{\mathrm{r}}$ of HV PMMA is around 0.5, for MV PMMA ranges between 0.5 and 0.4 , and for LV PMMA is between 0.4 and 0.3 . Those differences between materials are attributed to their different rheological properties as it was previously studied. ${ }^{[14]}$

Differences between OSD and TSD have been up to now studied. Now, the influence of process parameters in TSD will be studied (experiments T1 vs T2 and T4 vs T5).

Figure 7 shows the changes observed in $N_{0}$, and $\varphi$ when modifying the $P_{1}$ for both foaming temperatures. Lines between points do not claim for a linear tendency, but they are included to allow easier visualization of the obtained results. Focusing on HV PMMA, a higher $P_{1}$ (Figure 2a) results in higher $N_{0}$ and smaller $\varphi$ independently on $T_{\mathrm{f}}$. On the other hand, when using $T_{\mathrm{f}}=100^{\circ} \mathrm{C}$ the cell nucleation density is higher, and the cell size is smaller.

Considering MV PMMA, the tendency with the residual pressure is the same when the foaming temperature is $50^{\circ} \mathrm{C}$, although when increasing the saturation temperature up to $100{ }^{\circ} \mathrm{C}$, the tendency is reversed. Thus, when foaming at $100{ }^{\circ} \mathrm{C}$, a higher residual pressure leads to smaller cell nucleation densities and slightly larger cell sizes.

For LV PMMA, this reversed tendency is the one controlling the behavior. So independently of working at $T_{\mathrm{f}}=50$ or $100^{\circ} \mathrm{C}$ and increase in $P_{1}$ leads to smaller cell nucleation densities and bigger cell sizes.

Concluding, the new proposed process leads to nanocellular materials free of defects, although the cell size is increased, and the cell nucleation density decreased in comparison with the standard one-step process. On the other hand, obtained results when changing the production parameters in the two-stages depressurization process, depend on the used polymeric matrix.

Foaming mechanisms are discussed in the next section to understand the obtained results.

\subsection{Discussion of the Foaming Mechanisms}

Differences obtained when changing the residual pressure are not easy to understand or to model. Classical nucleation Theory (CNT) has been proved to not correctly model the experimental results obtained when the cell size is on the nanometric scale. However, scarce bibliography can be found dealing with this topic. For this reason, in this work, a discussion regarding foaming mechanisms has been established, aiming to advance in the understanding of foaming mechanisms in nanocellular polymers. (a)

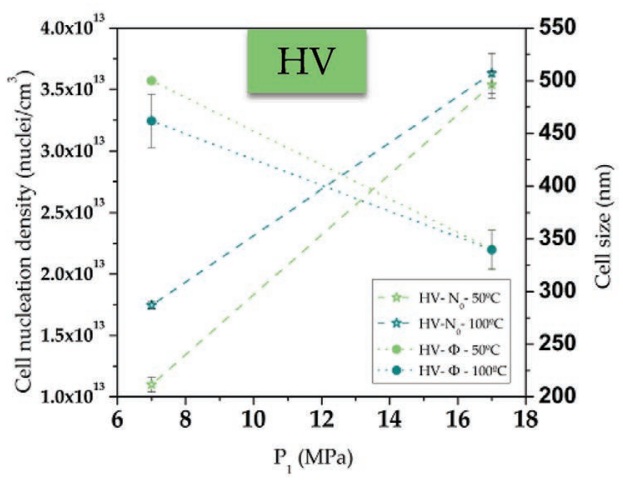

(b)

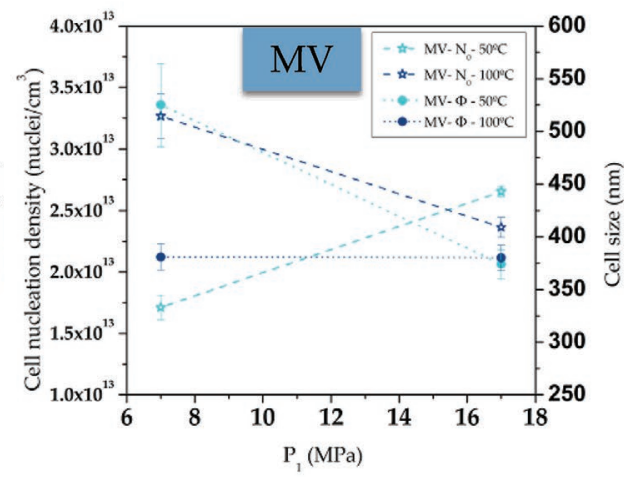

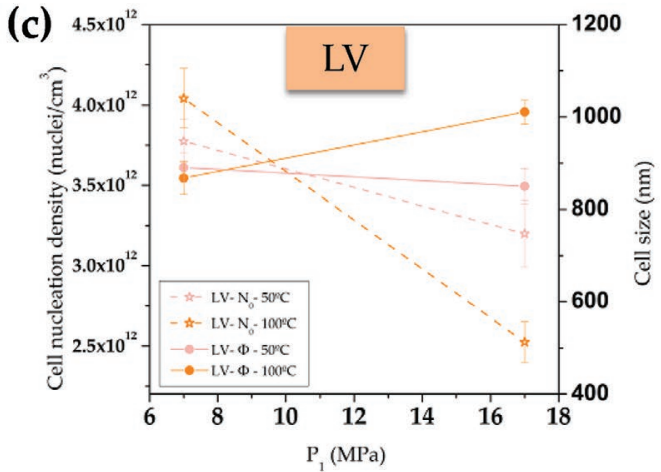

Figure 7. Change in the cell nucleation density and the cell size as a function of the residual pressure, for a) HV PMMA, b) MV PMMA, and c) LV PMMA. 
In order to discuss the obtained results, an improved model developed from the CNT will be used (see Section S1 in the Supporting Information). The proposed explanations of the foaming mechanisms given herein are based on observation, thus further experiments are needed to close the discussion and establish a complete theoretical model on the foaming of nanocellular polymers.

\subsubsection{Responsible Mechanisms of Changes in the Nanocellular Structure}

From One-Step Depressurization to the Two-Stage Depressurization Foaming Process: The first point is to understand when nucleation and growth occurs. As explained in Section 2.3, in one-step foaming, both mechanisms take place simultaneously when the pressure is released. In the case of a two-stage depressurization process, this situation is believed to be quite different.

First of all, when increasing the temperature from $T_{\text {sat }}$ to $T_{\mathrm{f}}$, a thermodynamic instability is created. Thus, nucleation and growth may take place. However, the effect of this increase is assumed to be much smaller than the one triggered by both depressurizations.

Additionally, any pressure drop leads to the creation of nucleation sites (see Section S1 in the Supporting Information); so in both depressurizations, nucleation sites are believed to be created. In our understanding of the process, it is theorized that after first depressurization the nucleus would not be able to fully grow due to the presence of the external pressure $P_{1}$. Herein, it is assumed that growth only remains as long as there exists a $\Delta P$, (difference between the gas pressure and the solid phase one). Figure 5 shows a homogeneous cellular structure of the final materials, there is not a second population of cells. This could mean that growth in this first depressurization should be small and negligible in comparison with that produced in the second depressurization. Cells created and growth in this second depressurization are indistinguishable from the ones created in the first depressurization.

So, it is herein assumed that in in the TSD foaming process of the paper, nuclei are produced in both pressure drops while growth takes place mainly in the second one, being not possible to distinguish the cells created in the first pressure drop to those created in the second one.

Taking this into consideration, differences in the internal cellular structure between both processes can now be analyzed. Figure 8 schematizes those differences.

Considering constant both the polymeric matrix and $T_{\mathrm{f}}$; $\Delta P, v$, and $D$ are the parameters influencing the final cellular structure.

Regarding pressure difference, $\Delta P$ in the OSD process is higher than any of the two pressures drops in the proposed new method. So according to Equation (2), due to the exponential dependence of nucleation with this parameter (see Section S1 in the Supporting Information), the pressure difference in single depressurization seems to lead to a higher $N_{0}$ independently on how $\Delta P_{1}$ and $\Delta P_{2}$ are distributed

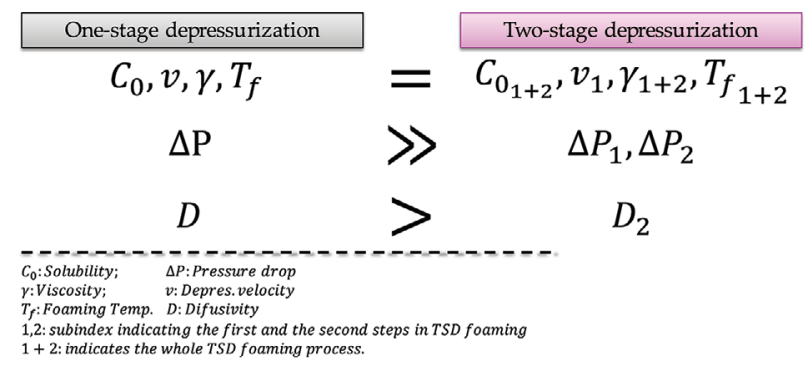

Figure 8. Scheme of differences between parameters influencing the cellular structure in one-depressurization and two-stage depressurization process.

$\Delta P>\Delta P_{1}, \Delta P_{2} \rightarrow \exp (\Delta P) \gg \exp \left(\Delta P_{1}\right)+\exp \left(\Delta P_{2}\right) \rightarrow N_{0} \gg N_{01}+N_{02}$

Conversely, considering the diffusivity right after depressurization and taking into account that it increases with pressure, this parameter is the only one negatively influencing nucleation in the OSD in comparison with the TSD process according to the proposed model.

So due to the strong dependence of cell nucleation density with the pressure drop, it seems that this is the primary mechanism contributing to the higher the cell nucleation density in the one-depressurization process. In conclusion, starting from the same saturation pressure, a two-stage depressurization reduces the number of nucleation sites with the consequent increase of the cell size.

In the same logic, an increase the number of nucleation sites in TSD can be achieved by increasing $\Delta P$, this can be done by using a higher $P_{\text {sat }}$ in TSD than in OSD (see Section S2 in the Supporting Information).

Differences Triggered by Changing the Residual Pressure: When working with the TSD process, the relation that it is believed to exist between the different processing parameters as a function of the residual pressure is shown schematically in Figure 9. All of this based on the experimental results. As it is shown in Table 2 and Figure 2, the first pressure drop is equivalent independently of the used residual pressure. Therefore, it could be assumed that the number of nucleation sites created in this first pressure drop and the produced growth is also comparable.

It is in the second part of the process, i.e., the second depressurization, when discrepancies are found. As it was previously commented, it is essential to consider that in this second depressurization, nucleation and growth are considered to be simultaneous processes. As can be seen in Figure 9, the pressure drop and the pressure drop rate are higher for higher residual pressure, contributing positively to the nucleation density. Conversely, in the moment of depressurization, the diffusivity has been assumed to be higher for higher residual pressure, ${ }^{[25]}$ so this reduces the number of nucleation sites. Additionally, the increase in the residual pressure, depressurization rate, and diffusivity leads to a rise in the cell growth rate (see Section S1 in the Supporting Information).

There exists a real competition between the previously mentioned parameters, pressure drop, depressurization velocity, and diffusivity, which also depends on the used material. In order to consider the contribution of each parameter, the proposed 


\begin{tabular}{|c|c|c|}
\hline High residual pressure & & Low residual pressure \\
\hline$C_{0_{H}}, \gamma_{H}, T_{f_{H}}, \Delta P_{1_{H}}$ & $=$ & $C_{0_{L}}, \gamma_{L}, T_{f_{L}}, \Delta P_{1_{L}}$ \\
\hline$\Delta \mathrm{P}_{2_{\mathrm{H}}}, v_{2_{H}}, \mathrm{D}_{2_{H}}$ & & $\Delta P_{2_{L}}, v_{2_{L}}, D_{2_{L}}$ \\
\hline
\end{tabular}

Figure 9. Scheme of differences between parameters influencing the cellular structure when changing the residual pressure in the two-stage foaming process. (Index $\mathrm{H}$ refers to high residual pressure and index $\mathrm{L}$ refers to low residual pressure.)

model introduces two parameters. The first one, $N_{\mathrm{p}}$, takes into account the competition between the depressurization velocity and the diffusivity, ${ }^{[17]}$ that as explained before, have opposite effects in nucleation density. The expression of this number is given by Equation (3)

$N_{\mathrm{p}}=\frac{r_{\mathrm{c}}^{2}}{D} \frac{\mathrm{d} P_{\mathrm{li}}}{\mathrm{d} t}$

That means that the depressurization velocity should by fast enough to compete with diffusivity and to prevent a fastergrowth of the influence volume (IV) that leads to a decrease in the nucleation rate. An increase in $N_{\mathrm{p}}$ implies a rise in $N_{0}$.

On the other hand, as previously commented, the viscosity of the gas/polymer system also plays a role. A high viscosity slows down the effect of the diffusivity, struggles against cell growth. ${ }^{[26-28]}$ If viscosity $(\mu)$ decreases, there is less resistance to cell growth, and the increment of IV mainly depends on the diffusivity (see Section S1 in the Supporting Information). This contribution is given by the Schmidt's number, ${ }^{[17]}$ the increment of which leads to an increase of $N_{0}$ (Equation (4))

$N_{\mathrm{Sc}}=\frac{\mu}{\rho D}$

In short, according to this model, the same pressure drop, depressurization velocity, and diffusivity will have a different effect on different polymers; this is consistent with the observed when studying the effect of the processing parameters in a TSD process in the three PMMA. ${ }^{[8]}$ Assuming a situation in which diffusivity increases, $N_{\mathrm{p}}$ will decrease, but a high enough

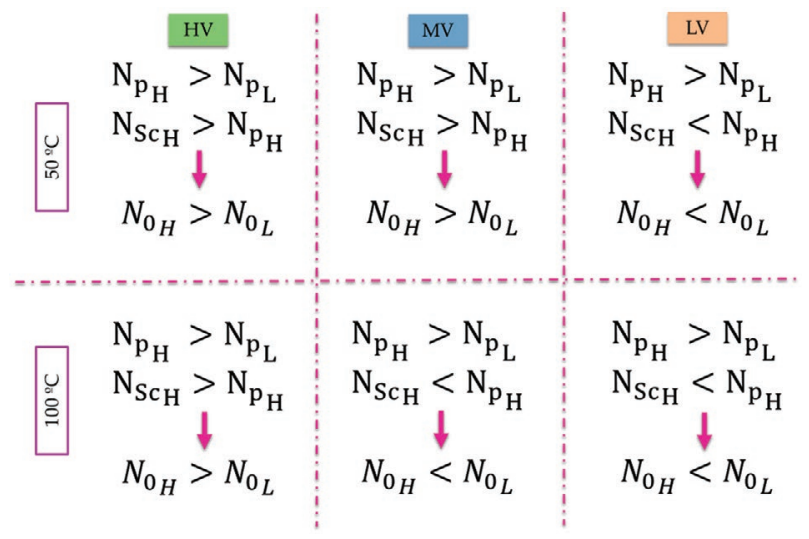

Figure 10. Scheme of the competition between $N_{\mathrm{p}}$ and $N_{\mathrm{Sc}}$ for the different materials and foaming conditions. viscosity will also lead to an increase of $N_{\mathrm{S}_{c}}$. Then, if $N_{\mathrm{S}_{c}}$ is higher than $N_{\mathrm{p}}$, the number of nucleation site will rise, and in the contrary situation $N_{0}$ would decrease.

Analyzing, in particular, the cases presented in this work, and according to the model in this work, the situations of Figure 10 could take place. For the highest viscous PMMA, a higher $P_{1}$ leads to higher cell nucleation density and smaller cell size (Figure 7). Meaning that $N_{\mathrm{pH}}>N_{\mathrm{pL}}$ for all the polymers due to this number only depends on the process parameters. Moreover, this result seems to indicate that the viscosity in this polymer is high enough to cancel out the effects of a higher diffusivity. So $N_{\mathrm{SCH}}>N_{\mathrm{pH}}$. Besides, a higher temperature leads to higher cell nucleation densities in this material (see Section S1 in the Supporting Information).

When working at $50^{\circ} \mathrm{C}$ with MV PMMA, this situation remains the same. However, increases foaming temperature up to $100^{\circ} \mathrm{C}$ is accompanied by a decrease in PMMA viscosity and an increase in diffusivity. So it seems that when increasing the foaming temperature, the Schmidt's number for the highest $P_{1}$ is no longer higher than $N_{\mathrm{p}}$ and, as a consequence, the situation reverts, cell density decreases when increasing the residual pressure.

Predictably, LV material, present such slow viscosity that even at $50{ }^{\circ} \mathrm{C}$ of foaming temperature $N_{\mathrm{SCH}}<N_{\mathrm{pH}}$. So, a smaller residual pressure seems to be better to enhance the nucleation in this polymer.

\subsubsection{Responsible Mechanisms for Defects Removal}

The origin for the removal of the defects in the multiple depressurization process could now be understood. The key reason it is believed to be the high gas pressure created in the cells during cell growth. In one-step foaming, the gas pressure is so high during the expansion of the material that the polymer is suddenly under enormous tension. Moreover, getting back to Figure 8, diffusivity is also higher in the one-step depressurization process. Meaning that the gas takes advantage of any initial defect of the polymer to be concentrated on that defect; this together with the fact that the polymer cannot withstand the high pressure if the viscosity of the polymer is not enough leads to cracks and defects, like those shown in Figure 4. Therefore, it could be that the combination of the presence of small defects during the production of the solid precursor (challenging to avoid when they are produced by compression molding) together with the high pressure developed in the material in the one-step foaming process are the key reasons promoting he presence of micrometric and millimetric defects in materials with low or medium viscosities.

For high viscosity materials, HV material, the viscosity seems to be high enough to prevent those big defects, while as viscosity decreases, there is not any barrier for defect formation.

With the proposed method, due to the double depressurization, it seems that the pressure of the gas while growing is smaller; this fact accompanied by a slower diffusivity, also promoted with the TSD process, leads to a smooth growth free of defects.

In conclusion, the two-stage depressurization foaming process leads to nanocellular materials free of defects, although there is a reduction of cell nucleation density that should be compensated by the use of different saturation parameters. 


\section{Conclusions}

A new production method for nanocellular materials is proposed in this work. Two-stage depressurization process modifies the depressurization step of the commonly used gas dissolution foaming process. This modification allows removing micrometric and millimetric defects appearing in some materials when foaming at certain conditions.

In this research, depressurization has been carried out in two stages, allowing to produce nanocellular PMMA free of defects from materials with different viscosities. Moreover, the obtained cellular structures were compared with those produced through a simple one-depressurization process. The new method leads to larger cell sizes and smaller cell nucleation densities. The analysis of the foaming mechanisms playing a role in this process has led to conclude that it could be that a lower pressure of gas when the nucleation sites start to grow is beneficial to avoid the generation of defects. On the other hand, in order to obtain comparable cellular structures with the ones obtained through the one-depressurization process, an increase in the saturation pressure is necessary to promote a higher pressure drop that could maximize nucleation.

On the other hand, modifications in the parameters of this new method were carried out, so the depressurization stages were modified. It can be concluded that the obtained results depend on the viscosity of the used polymer. Higher residual pressures are beneficial for polymers presenting a high viscosity, while for those with lower viscosity, a lower residual pressure enhances the nucleation density.

\section{Supporting Information}

Supporting Information is available from the Wiley Online Library or from the author.

\section{Acknowledgements}

Financial assistance from the Junta of Castile and Leon (VA275P18) and Spanish Ministry of Science, Innovation and Universities (RTI2018098749-B-100) and the "Ente Público Regional de la Energía de Castilla y León" (EREN) are gratefully acknowledged. Financial support from FPU grant FPU14/02050 (V.B.) from the Spanish Ministry of Education and Junta of Castile and Leon grant (J.M.-d.L.) are gratefully acknowledged.

\section{Conflict of Interest}

The authors declare no conflict of interest.

\section{Keywords}

nanocellular free of defects, nanocellular polymethylmethacrylate, one step foaming

Received: April 24, 2020

Revised: July 13, 2020

Published online: August 11, 2020

[1] S. Costeux, J. Appl. Polym. Sci. 2014, 131, 41293.

[2] B. Notario, J. Pinto, M. A. Rodriguez-Perez, Polymer 2015, 63, 116.

[3] M. Di Luccio, R. Nobrega, C. Borges, Polymer 2000, 41, 4309.

[4] S. Walheim, R. S. Bradley, P. D. Jones, E. Routledge, Science 2009, $520,520$.

[5] O. Ramström, R. J. Ansell, Chirality 1998, 10, 195.

[6] B. Gates, Y. Yin, Y. Xia, Chem. Mater. 1999, 11, 2827.

[7] F. M. Menger, T. Tsuno, G. S. Hammond, J. Am. Chem. Soc. 1990, 112, 1263

[8] C. Du Fresne Von Hohenesche, D. F. Schmidt, V. Schädler, Chem. Mater. 2008, 20, 6124.

[9] A. S. Zalusky, R. Olayo-Valles, J. H. Wolf, M. A. Hillmyer, J. Am. Chem. Soc. 2002, 124, 12761.

[10] H. Guo, A. Nicolae, V. Kumar, Cell. Polym. 2016, 35, 119.

[11] H. Guo, Ph.D. Thesis, University of Washington 2015.

[12] J. Martín-de León, V. Bernardo, M. Rodríguez-Pérez, Materials 2019, $12,797$.

[13] S. Costeux, L. Zhu, Polymer 2013, 54, 2785.

[14] J. Martín-de Leon, V. Bernardo, E. Laguna-gutiérrez, M. Á. Rodríguez-pérez, Polym. Int. 2020, 69, 72.

[15] R. Dugad, G. Radhakrishna, A. Gandhi, Cell. Polym. 2019, 39, 141.

[16] F. Farhanmoghaddam, A. Javadi, Cell. Polym. 2020, 39, 172.

[17] S. Costeux, I. Khan, S. P. Bunker, H. K. Jeon, J. Cell. Plast. 2015, 51, 197.

[18] L. Urbanczyk, C. Calberg, C. Detrembleur, C. Jérôme, M. Alexandre, Polymer 2010, 51, 3520.

[19] H. Guo, V. Kumar, Polymer 2015, 56, 46.

[20] H. Guo, V. Kumar, Polymer 2015, 57, 157.

[21] J. Martín-de León, V. Bernardo, M. Rodríguez-Pérez, Polymers 2016, 8, 265.

[22] E. Laguna-Gutierrez, R. Van Hooghten, P. Moldenaers, M. Angel Rodriguez-Perez, J. Appl. Polym. Sci. 2015, 132, 42430.

[23] J. Pinto, E. Solorzano, M. a. Rodriguez-Perez, J. A. de Saja, J. Cell. Plast. 2013, 49, 555.

[24] V. Kumar, N. P. Suh, Polym. Eng. Sci. 1990, 30, 1323.

[25] J. Crank, The Mathematics of Diffusion, Oxford University Press, Oxford 1975

[26] J. M. de León, V. Bernardo, E. Laguna-Gutiérrez, M. Á. RodríguezPérez, Polym. Int. 2020, 69, 72.

[27] S. Costeux, M. H. Jeon, T. S. Bunker, I. Khan, in FOAMS 2012 Conf., 2012, p. 1.

[28] C. Forest, P. Chaumont, P. Cassagnau, B. Swoboda, P. Sonntag, Polymer 2015, 77, 1. 